\title{
Efficacy of denosumab therapy for neurofibromatosis type I with osteoporosis and history of fractures: a case report
}

This article was published in the following Dove Press journal:

Therapeutics and Clinical Risk Management

\author{
Masashi Uehara' \\ Yukio Nakamura' \\ Jun Takahashi' \\ Mikio Kamimura ${ }^{2}$ \\ Fumihiro Isobe' \\ Tomomi Yamaguchi ${ }^{3,4}$ \\ Tomoki Kosho 3,4 \\ Shigeharu Uchiyama ${ }^{1,5}$ \\ Takako Suzuki' \\ Hiroyuki Kato' \\ 'Department of Orthopedic Surgery, \\ Shinshu University School of \\ Medicine, Asahi, Matsumoto, Japan; \\ ${ }^{2}$ Center for Osteoporosis and Spinal \\ Disorders, Kamimura Orthopedic \\ Clinic, Kotobuki, Matsumoto, Japan; \\ ${ }^{3}$ Department of Medical Genetics, \\ Shinshu University School of \\ Medicine, Asahi, Matsumoto, Japan; \\ ${ }^{4}$ Center for Medical Genetics, Shinshu \\ University Hospital, Asahi, Matsumoto, \\ Japan; ${ }^{5}$ Department of Orthopedic \\ Surgery, Okaya City Hospital, \\ Honmachi, Okaya, Japan
}

Correspondence: Yukio Nakamura

Department of Orthopedic Surgery, Shinshu University School of Medicine,

Asahi 3-I-I, Matsumoto 390-862I, Japan

Tel +8I 263372659

Fax +8I 263358844

Email yxn I4@aol.jp
Background: The natural history and pathogenesis of the skeletal abnormalities found in neurofibromatosis type 1 (NF1) are poorly understood, and the therapeutic options for these manifestations remain limited. This report first describes the clinical outcomes of denosumab treatment for a patient with NF1 suffering from osteoporosis.

Methods: We enrolled a patient with NF1 under denosumab treatment for osteoporosis, prior fractures, and no improvement in bone mineral density (BMD) over 3 years of alendronate therapy. BMD was monitored by dual-energy X-ray absorptiometry. Tested laboratory data included bone-specific alkaline phosphatase, urinary type I collagen amino-terminal telopeptide, tartrate-resistant acid phosphatase 5b, 1-alpha, 25-dihydroxyvitamin $\mathrm{D}_{3}$, and parathyroid hormone. BMD and laboratory data were evaluated before, between 2 and 4 months, and at $6,12,18$, and 24 months of treatment.

Case presentation: During 2 years of denosumab therapy for osteoporosis in a 58 -year-old female NF1 patient with prior fractures, BMD increased by $6.5 \%$ in the lumbar spine and $10.6 \%$ in the total hips, and bone turnover markers were notably improved. No fractures occurred during the latter half of treatment.

Conclusion: Denosumab represents an effective treatment option for osteoporosis in NF1 patients.

Keywords: denosumab, fracture, neurofibromatosis type 1, osteoporosis

\section{Introduction}

With an incidence of 1 in 3,000-4,000 individuals, neurofibromatosis type 1 (NF1) is a haploinsufficient and multisystemic disease caused by inherited or sporadic mutations in the NF1 gene. The pathoetiology of the multiple manifestations found in this disease across different organ systems is complex. ${ }^{1}$ NF1 has an autosomal dominant pattern of inheritance, high clinical variability, complete penetrance, and age-dependent complications. ${ }^{2}$ In addition to neurofibromas, there are numerous other clinical manifestations in NF1, such as gliomas, peripheral nerve sheath tumors, and other malignant tumors as well as nontumor effects that include skeletal dysplasia and learning disabilities. The diagnosis of NF1 has been established on the basis of clinical criteria and genetic testing. ${ }^{3}$ However, the natural history and pathogenesis of the skeletal abnormalities in this disorder are poorly understood, and therapeutic options currently remain limited. Some manifestations, such as low bone mass, short stature, macrocephaly, long bone dysplasia, and vertebral defects, can lead to clinically significant consequences. ${ }^{4}$ 
High parathyroid hormone and low 25-hydroxyvitamin D values are generally observed in patients with NF1 and may adversely affect bone mass and fracture risk. ${ }^{5-7}$ In vitro studies have revealed increased C-terminal telopeptide of type 1 collagen (CTX) and type I procollagen N-terminal propeptide (PINP) in NF1 patients as well. ${ }^{8}$ To date, reports on the treatment of patients with NF1 and accompanying osteoporosis are scarce. Heervä et $\mathrm{al}^{9}$ described that weekly oral alendronate (ALN) with daily vitamin D substantially, but not significantly, increased bone mineral density (BMD) levels. They also showed that values of CTX and PINP became reduced but that of serum tartrate-resistant acid phosphatase 5b (TRACP-5b) was unchanged, whereas previous in vitro studies demonstrated that NF1 osteoclasts displayed insensitivity to the apoptotic signals caused by bisphosphonates (BPs). On the other hand, Benlidayi et al ${ }^{10}$ recently found that weekly oral doses of the BP risedronate (RIS) significantly increased both lumbar and hip bone mass during the first year of therapy and proposed RIS as a potential therapy for osteoporosis complicating NF1. However, it remains controversial which osteoporotic treatment is optimal for osteoporotic NF1 patients.

Denosumab is an anti-receptor activator of nuclear factor_ $\kappa \mathrm{B}$ ligand (RANKL) monoclonal antibody approved for the treatment of osteoporosis and prevention of skeletal metastatic complications. ${ }^{11}$ To date, there are no published accounts of denosumab treatment for NF1 complicated with osteoporosis.

In this report, we describe the clinical results of a 58-year-old woman with NF1 accompanied by osteoporosis and a history of fractures. Improvements in BMD and bone metabolic markers were observed over 2 years of denosumab treatment.

\section{Case presentation}

A 58-year-old woman with NF1 was referred to our department for treatment. Her BMD and laboratory data are presented in Tables 1 and 2, respectively. She had not exhibited any clinical disease signs at birth, but dermatological examination of a café au lait spot during the upper grades of elementary school disclosed NF1. Genetic testing confirmed a deletion mutation in NF1 (c.6525delT) causing an amino acid substitution at position 2175 (p.2175fs). She later required resection of neurofibromas around her back and breast in her 40s. Foot pain appeared at age 55, at which time a fatigue fracture was discovered during a visit to an orthopedic clinic. She was referred to the Endocrinology and Metabolism Department of our institution under suspicion of osteoporosis and began conservative treatment for hyperparathyroidism. Osteoporotic treatment was commenced for the patient's diminished lumbar and proximal femur BMD values due to past fragility fractures. She had been receiving ALN for the previous 3 years at a nearby clinic. Despite the ALN administration, the bone turnover marker values remained high values. As BMD values had remained substantially low, we opted for denosumab owing to its reported effectiveness following BP therapy. ${ }^{12}$

Denosumab was injected subcutaneously every 6 months (ie, at 0, 6, 12, 18, and 24 months) and bone metabolism marker and BMD values were examined before, between 2 and 4 months, and at 6, 12, 18, and 24 months of treatment. Serum TRACP-5b and urinary type I collagen amino-terminal telopeptide (NTX) greatly decreased after the first administration. Despite the denosumab administration, bone turnover markers were remarkably increased to the 18 months after the therapy. At the study end point, the bone turnover markers were greatly inhibited.

At 1 year of denosumab treatment, her lumbar BMD had not increased and she had experienced a vertebral fracture. Around that time, her activity had started to decline and she required the use of a wheelchair. BMD began to fall. At 2 years of denosumab treatment, however, her lumbar BMD T-score was improved from -2.8 to -2.4 and her total hip BMD T-score was ameliorated from -3.9 to -3.5 . The percent increases in BMD were $6.5 \%$ for the lumbar spine and $10.6 \%$ for total hips at the study end point.

This study was approved by the institutional ethical review board of Shinshu University School of Medicine (approval number: 2365) prior to its start and was conducted in accordance with the ethical standards set forth in the

Table I Patient characteristics and lumbar and total hip BMD before and at 6, 12, I8, and 24 months of denosumab therapy

\begin{tabular}{lllllllll}
\hline Parameters & $\mathbf{- 2 2}$ months & $\mathbf{- 1 4}$ months & $\mathbf{- 3}$ months & $\mathbf{0}$ month & $\mathbf{6}$ months & $\mathbf{1 2}$ months & 18 months & $\mathbf{2 4}$ months \\
\hline Medication & ALN & ALN & ALN & Denosumab & Denosumab & Denosumab & Denosumab & Denosumab \\
Lumbar BMD $\left(\mathrm{g} / \mathrm{cm}^{2}\right)$ & 0.77 & 0.69 & 0.79 & 0.77 & 0.78 & 0.70 & 0.76 & 0.82 \\
Lumbar T-score & -2.8 & -2.9 & -2.6 & -2.8 & -2.7 & -3.4 & -2.9 & -2.4 \\
Total hip BMD $\left(\mathrm{g} / \mathrm{cm}^{2}\right)$ & 0.52 & 0.48 & 0.53 & 0.47 & 0.52 & 0.53 & 0.54 & 0.52 \\
Total hip T-score & -3.5 & -3.5 & -3.4 & -3.9 & -3.5 & -3.3 & -3.3 & -3.5 \\
\hline
\end{tabular}

Abbreviations: ALN, alendronate; BMD, bone mineral density. 
Table 2 Serum Alb, Ca, bone turnover markers, I,25(OH) 2 , and whole PTH values before and at I, 3, 6, I2, I8, and 24 months of denosumab therapy

\begin{tabular}{|c|c|c|c|c|c|c|c|c|c|}
\hline Parameters & -22 months & -12 months & 0 month & I month & 3 months & 6 months & 12 months & 18 months & 24 months \\
\hline Medication & ALN & ALN & Denosumab & Denosumab & Denosumab & Denosumab & Denosumab & Denosumab & Denosumab \\
\hline Alb-corrected & 8.8 & 9.1 & 9.1 & 8.2 & 8.5 & 8.6 & 9.3 & 9.5 & 8.6 \\
\hline \multicolumn{10}{|l|}{$\mathrm{Ca}(\mathrm{mg} / \mathrm{dL})$} \\
\hline $\operatorname{BAP}(\mu g / L)$ & 76.6 & 79.5 & 59.6 & 54.2 & 36.2 & 79.8 & 56.3 & 53.8 & 15.4 \\
\hline PINP (ng/mL) & $\mathrm{N} / \mathrm{A}$ & N/A & 66.6 & 34.4 & 37.2 & 88.8 & 79.3 & 114 & 26.4 \\
\hline NTX & $\mathrm{N} / \mathrm{A}$ & 61.8 & 55.9 & 8.3 & 12.9 & 51.8 & 92 & 198 & 9.8 \\
\hline \multicolumn{10}{|l|}{ (nmolBCE/mmol/CRE) } \\
\hline TRACP-5b (mU/dL) & N/A & N/A & 688 & 83 & 84 & 355 & 775 & $\mathrm{I}, 070$ & 150 \\
\hline $\mathrm{I}, 25(\mathrm{OH})_{2} \mathrm{D}(\mathrm{pg} / \mathrm{mL})$ & 44.4 & 50.7 & 36.6 & 76.5 & 58.2 & 25.9 & 32.2 & 107 & 93.5 \\
\hline Whole PTH (pg/mL) & $\mathrm{N} / \mathrm{A}$ & 61.9 & 73.2 & 187 & 150 & 70.9 & 72.4 & 68.1 & 270 \\
\hline
\end{tabular}

Abbreviations: ALN, alendronate; Alb, albumin; Ca, calcium; I,25(OH) $\mathrm{D}$, I-alpha, 25-dihydroxyvitamin $\mathrm{D}_{3}$; PTH, parathyroid hormone; BAP, bone-specific alkaline phosphatase; PINP, type I procollagen N-terminal propeptide; NTX, type I collagen amino-terminal telopeptide; TRACP-5b, tartrate-resistant acid phosphatase 5b; N/A, not available.

1964 Declaration of Helsinki for research involving human subjects. Written informed consent was obtained from the patient for publication prior to this study.

\section{Discussion}

This report evaluates the clinical outcomes of a patient with NF1 receiving denosumab for complicating osteoporosis. At 2 years of treatment, lumbar and total hip BMD values had increased by $6.5 \%$ and $10.6 \%$, respectively. No fractures occurred during the second half of therapy, and her bone metabolism markers were notably improved. To our knowledge, this study is the first of its kind describing the successful osteoporotic treatment of a BP-unresponsive NF1 patient with a history of fractures.

Several BPs and other antiresorptive agents have been employed to reduce the increased bone turnover contributing to osteoporosis in NF1. ${ }^{9,10}$ For primary osteoporosis, 5 years is considered by many as a suitable time point to evaluate the effect and merit of continuing a BP preparation for fracture suppression. ${ }^{13}$ Despite a lack of consensus on the treatment of osteoporosis in NF1, BPs may currently be a good choice. ${ }^{9110}$ Also inhibiting bone resorption, denosumab is a strong candidate for BMD improvement in BPunresponsive cases, ${ }^{12}$ although there have been no reports on denosumab for NF1 and osteoporosis. In our patient, BMD values did not substantially change and she broke her foot during 3 years of ALN treatment. As a result, we switched to denosumab to prevent ensuing fractures and improve BMD after careful discussion with her and her family. In this case, bone turnover markers were increased prior to the denosumab therapy regardless of BP administration. Also, the decreased bone turnover markers were greatly increased after the denosumab therapy. It is considered that these findings could be caused by fragility fractures.
At 1 year of denosumab treatment, her lumbar BMD had not increased and she had suffered a vertebral fracture due to a decline in activity. However, due to the strong inhibition of the increase of bone turnover markers caused by fractures during BP and denosumab therapy, both lumbar and total hip BMD values became increased at 2 years of treatment, with no additional fractures in the latter half of the study. At 2 years of treatment, lumbar and total hip BMD values had increased by $6.5 \%$ and $10.6 \%$, respectively.

Bone resorption markers, such as urinary NTX and TRACP-5b, and bone formation markers, including PINP and BAP, are often used to monitor the effects of osteoporosis treatment. ${ }^{14}$ Despite the administration of antiresorption drugs, the increased bone turnover markers were greatly inhibited at 24 months of therapy. Also, the 2-yeardenosumab treatment substantially increased BMD. These results indicated that denosumab strongly suppressed bone resorption in NF1 patient's accompanying osteoporosis and presumably produced her gains in bone density.

\section{Conclusion}

Two years of denosumab treatment improved BMD values and bone turnover markers in an NF1 patient with osteoporosis. The drug therefore represents an effective treatment option in such cases and warrants further study.

\section{Disclosure}

The authors report no conflicts of interest in this work.

\section{References}

1. Dunning-Davies BM, Parker A. Annual review of children with neurofibromatosis type 1. Arch Dis Child Educ Pract Ed. 2016;101:102-111.

2. Gómez M, Batista O. Molecular diagnosis as a strategy for differential diagnosis and at early ages of neurofibromatosis type 1 (NF1). Rev Med Chil. 2015;143(10):1320-1330. 
3. Korf BR. Diagnosis and management of neurofibromatosis type 1 . Curr Neurol Neurosci Rep. 2001;1(2):162-167.

4. Takahara K, Kamimura M, Nakagawa H, Uchiyama S. Changes in biochemical markers of bone in patients with insufficiency fractures. $J$ Bone Miner Metab. 2004;22:618-625.

5. Brunetti-Pierri N, Doty SB, Hicks J, et al. Generalized metabolic bone disease in neurofibromatosis type I. Mol Genet Metab. 2008;94(1): $105-111$.

6. Tucker T, Schnabel C, Hartmann M, et al. Bone health and fracture rate in individuals with neurofibromatosis 1 (NF1). J Med Genet. 2009;46(4): 259-265.

7. Petramala L, Giustini S, Zinnamosca L, et al. Bone mineral metabolism in patients with neurofibromatosis type 1 (von Recklingausen disease). Arch Dermatol Res. 2012;304(4):325-331.

8. Heervä E, Peltonen S, Svedström E, Aro HT, Väänänen K, Peltonen J. Osteoclasts derived from patients with neurofibromatosis 1 (NF1) display insensitivity to bisphosphonates in vitro. Bone. 2012;50(3):798-803.

9. Heervä E, Huilaja L, Leinonen P, Peltonen S, Peltonen J. Follow-up of six patients with neurofibromatosis 1-related osteoporosis treated with alendronate for 23 months. Calcif Tissue Int. 2014;94(6):608-612.
10. Benlidayi IC, Ortac EA, Kozanoglu E. Risedronate therapy for neurofibromatosis type 1-related low bone mass: a stitch in time saves nine. Acta Clin Belg. 2015;70(2):130-132.

11. Diédhiou D, Cuny T, Sarr A, Norou Diop S, Klein M, Weryha G. Efficacy and safety of denosumab for the treatment of osteoporosis: a systematic review. Ann Endocrinol (Paris). 2015;76(6):650-657.

12. Kamimura M, Nakamura Y, Ikegami S, Uchiyama S, Kato H, Taguchi A. Significant improvement of bone mineral density and bone turnover markers by denosumab therapy in bisphosphonateunresponsive patients. Osteoporos Int. 2017;28:559-566.

13. Whitaker M, Guo J, Kehoe T, Benson G. Bisphosphonates for osteoporosis: where do we go from here? N Engl J Med. 2012; 366(22):2048-2051.

14. Vasikaran $\mathrm{S}$, Eastell R, Bruyère $\mathrm{O}$, et al. Markers of bone turnover for the prediction of fracture risk and monitoring of osteoporosis treatment: a need for international reference standards. Osteoporos Int. 2011; 22(2):391-420.
Therapeutics and Clinical Risk Management

\section{Publish your work in this journal}

Therapeutics and Clinical Risk Management is an international, peerreviewed journal of clinical therapeutics and risk management, focusing on concise rapid reporting of clinical studies in all therapeutic areas, outcomes, safety, and programs for the effective, safe, and sustained use of medicines. This journal is indexed on PubMed Central, CAS,

\section{Dovepress}

EMBase, Scopus and the Elsevier Bibliographic databases. The manuscript management system is completely online and includes a very quick and fair peer-review system, which is all easy to use. Visit $\mathrm{http}: / / \mathrm{www}$.dovepress.com/testimonials.php to read real quotes from published authors. 